\title{
Evolución de las condiciones de accesibilidad media global ofrecida por la red de infraestructuras del transporte en Manizales - Colombia
}

\author{
Diego A. Escobar ${ }^{*}$, Santiago Ruiz ${ }^{2}$ y Carlos A. Moncada ${ }^{3}$ \\ (1) Universidad Nacional de Colombia, Sede Manizales. Facultad de Ingeniería y Arquitectura, Departamento de \\ Ingeniería Civil, Grupo de investigación en Movilidad Sostenible. Campus La Nubia, Manizales, 170003, Colombia. \\ (correo-e: daescobarga@unal.edu.co) \\ (2) Universidad Nacional de Colombia, Sede Manizales. Facultad de Ingeniería y Arquitectura, Departamento de \\ Ingeniería Industrial, Grupo de Investigación en Innovación y Desarrollo Tecnológico. Manizales, 170004, Colombia. \\ (correo-e: sruizhe@unal.edu.co) \\ (3) Universidad Nacional de Colombia, Sede Bogotá. Facultad de Ingeniería, Departamento de Ingeniería Civil y \\ Agrícola, Programa de Investigación en Tránsito y Transporte - PIT. Ciudad Universitaria, Bogotá, 111321, Colombia. \\ (correo-e: camoncadaa@unal.edu.co)
}

* Autor a quien debe ser dirigida la correspondencia

Recibido Dic. 22, 2019; Aceptado Feb. 26, 2020; Versión final Abr. 15, 2020, Publicado Dic. 2020

\section{Resumen}

En esta investigación, se calculan, analizan y comparan las condiciones de accesibilidad territorial a nivel global de la conurbación Manizales - Villamaría entre los años 2010 y 2017. Se define el año 2010 como el escenario base para el cálculo de accesibilidad (Escenario 1) y se compara con la accesibilidad del año 2017 a partir de dos redes de trabajo: 1) uno académico y abordado por el grupo de investigación en movilidad sostenible (Escenario 2), y 2) una consultoría contratada por el municipio de Manizales (Escenario 3). Los resultados muestran que el escenario 2 propone intervenir prioritariamente el sector occidente y el escenario 3 propone intervenir el sector oriente. La comparación de los escenarios 1 y 2 se consideran mucho más reales y consecuentes que los obtenidos al comparar los escenarios 1 y 3 . Se concluye que tener resultados poco reales puede direccionar los esfuerzos infraestructurales en sentidos posiblemente erróneos, y en este caso en particular, en sentido opuesto.

Palabras clave: accesibilidad; auditoria; comparación; gradiente; red vial

\section{Evolution of global average accessibility conditions offered by the transport infrastructure network in Manizales - Colombia}

\begin{abstract}
The objective of this research study is to calculate, analyze, and compare at the global level the conditions of territorial accessibility of the Manizales - Villamaría conurbation between the years 2010 and 2017. The year 2010 is defined as the base scenario (scenario 1) for accessibility calculations and is compared to the year 2017 by two different groups: 1) an academic group composed by the sustainable mobility research group (scenario 2) and 2) a private consultant group contracted by the municipality of Manizales (scenario 3 ). The results show that the scenario 2 proposes to intervene the west sector while the scenario 3 proposes to intervene the east sector. The results of comparing scenarios 1 and 2 are considered more realistic and consequential than those obtained when comparing scenarios 1 and 3 . It is concluded that having unrealistic results may put infrastructure efforts in the wrong direction, and in this particular case, in the opposite direction.
\end{abstract}

Keywords: accessibility; audit; comparison; gradient; road network 


\section{INTRODUCCIÓN}

Los modelos de desarrollo y crecimiento de una ciudad, se encuentran estrechamente ligados a la capacidad de implementar la infraestructura de servicios básicos requerida, teniendo en cuenta agua, electricidad, alcantarillado, vías, entre otras (Gonzales et al., 2007); con el fin de incrementar la productividad y minimizar la brecha económica entre miembros de la misma sociedad, en otras palabras, una adecuada inversión en infraestructura facilita y estimula el crecimiento económico de una sociedad (Urrunaga y Aparicio, 2012). Es mundialmente conocido que los recursos económicos que soportan las condiciones de bienestar de las comunidades no se distribuyen uniformemente alrededor del mundo (Weiss et al., 2018) y menos si se habla en términos de zonas urbanas y rurales. Considerando lo anterior, se tiene que las redes de infraestructura del transporte son uno de los pilares fundamentales de desarrollo, pues son el eje sobre el cual se expanden las ciudades $\mathrm{y}$, por lo tanto, se hace evidente la necesidad de una continua evaluación del sistema, con el objetivo de observar su cobertura, así como la definición de posibles zonas de intervención.

Hoy en día se analizan, de forma puntual, variables geográficas que permiten establecer las condiciones de accesibilidad a los servicios y oportunidades que las ciudades ofrecen, permitiendo identificar los verdaderos obstáculos que existen para mejorar la calidad de vida de las personas y de forma general, para mejorar sus condiciones de desarrollo como comunidad. Por otra parte, se han definido los llamados Nodos de Actividad Primaria - NAP (salud, educación, recreación y seguridad) como aquellos que sustentan las principales necesidades de una población en particular, que hacen parte del diario transcurrir de los habitantes y que influencian de forma directa las condiciones de sostenibilidad de una ciudad pues su importancia radica precisamente en la necesidad ciudadana de acceder a los mismos (Escobar et al., 2015a). La primera definición técnica del concepto "accesibilidad", se remonta hacia los años 50, en la cual Hansen lo define como el potencial de oportunidades para la interacción (Hansen, 1959); sin embargo, este concepto se ha utilizado desde la segunda década del siglo XX (Batty, 2009), aplicado en el campo de le economía. Ahora bien, en el marco de la agenda de equidad, establecida por los Objetivos de Desarrollo Sostenible (ODS) de las Naciones Unidas, se hace uso del concepto accesibilidad, pues a través de éste se respaldan las diferentes medidas de equidad (Weiss et al., 2018). Otras definiciones del término plantean la interacción entre formas básicas de actividad humana (Geurs y Van Bee, 2004), analizadas desde diferentes enfoques como: desarrollo orientado al tránsito (Conesa, 2018), estructura urbana (Boarnet y Wang, 2019), usos del suelo, inequidad (Bocarejo, 2012), planeación del transporte (Geurs, y Van Bee, 2004; Escobar et al., 2015b), influencia en el costo del transporte (Cui y Levinson, 2018), sostenibilidad (Vega, 2011; Escobar et al., 2015a), acceso a servicios u oportunidades (Wachs y Kumagai, 1973, Escobar et al., 2017, Sahebgharani et al., 2019), comercio (Zuluaga y Escobar, 2016; Montoya et al., 2017), Salud (Sarmiento et al., 2020), entre otros.

La ciudad de Manizales (capital del Departamento de Caldas) está localizada a una altura de $2150 \mathrm{msnm}$ bajo el seno de la cordillera central en los $5^{\circ} 3^{\prime} 58^{\prime \prime}$ de latitud Norte y $75^{\circ} 29^{\prime} 05^{\prime \prime}$ de longitud Oeste dentro del denominado eje cafetero colombiano (Figura 1); dada su ubicación, la ciudad presenta una topografía abrupta, con dificultades en sus procesos de urbanización que, en algunos casos, ha obligado al desarrollo de viviendas informales en zonas periféricas del municipio. Según datos oficiales, Manizales se extiende a lo largo de $572 \mathrm{~km}^{2}$, de los cuales $35,11 \mathrm{~km}^{2}(6,14 \%)$ corresponden a su área urbana, alojando a 371.345 habitantes; por otra parte, la ciudad se encuentra conurbada con el municipio vecino de Villamaría, el cual se extiende a lo largo de $461 \mathrm{~km}^{2}$, la zona urbana representa solo el 0,48\%, albergando una población de 48.625 habitantes (Alcaldía de Manizales, 2017). En relación a las condiciones de movilidad del área conurbada, se presentan un total de 783.504 viajes diarios (Alcaldía de Manizales, 2017), de los cuales, un $31 \%$ se realiza en modos de transporte sostenible ( $30,0 \%$ caminata, $0,7 \%$ bicicletas y $0,4 \%$ cable aéreo), un $36,9 \%$ se realiza en transporte público colectivo e individual $(31,3 \%$ buses y $5,6 \%$ taxi) y un $32,1 \%$ se realiza en transporte privado ( $20,6 \%$ vehículos y $11,5 \%$ motocicletas); adicionalmente el $17 \%$ de la población percibe que sus viajes actuales toman más tiempo que en años anteriores, según la encuesta de calidad de vida del año 2019 en Manizales. La conexión entre ambos municipios se realiza a través de una vía pavimentada de $4 \mathrm{~km}$. de longitud, siendo ésta la única alternativa existente, en términos de red vial. Frente a otras ciudades intermedias, el área conurbada de estudio, presenta un atraso en infraestructura vial, unas (o algunas) de las causas son la empinada topografía, la arquitectura propia del centro histórico, el aumento del parque automotor, diferentes tipos de vehículos en circulación, implementación de ciclorrutas y bulevares en las vías existentes lo que afecta directamente las condiciones operativas de la movilidad; todas las anteriores situaciones, contribuyen a que las condiciones de accesibilidad, en especial en los sectores periféricos de la ciudad, se vean diariamente afectadas y se refieran deficiencias.

A nivel global se han realizado importantes esfuerzos para medir con mayor precisión las condiciones de accesibilidad (Weiss et al., 2018), mediante el uso de bases de datos complejas (Long et al., 2018), con el fin de mejorar la métrica que pueda servir como base en el diseño e implementación de políticas de desarrollo urbano situación que se relaciona directamente con las posibilidades reales de respaldar la agenda de equidad propuesta por los ODS. Ahora bien, la principal característica operativa de la red de infraestructuras 
del transporte, que debe ser tenida en cuenta para el cálculo de la accesibilidad, es la velocidad (Liu y Zhu, 2004), encontrando que la estructura interna de una red, contribuye de forma directa a la percepción sobre del tiempo medio de viaje (Carrion y Lenvinson, 2019), lo cual involucra intrínsecamente la variable velocidad.

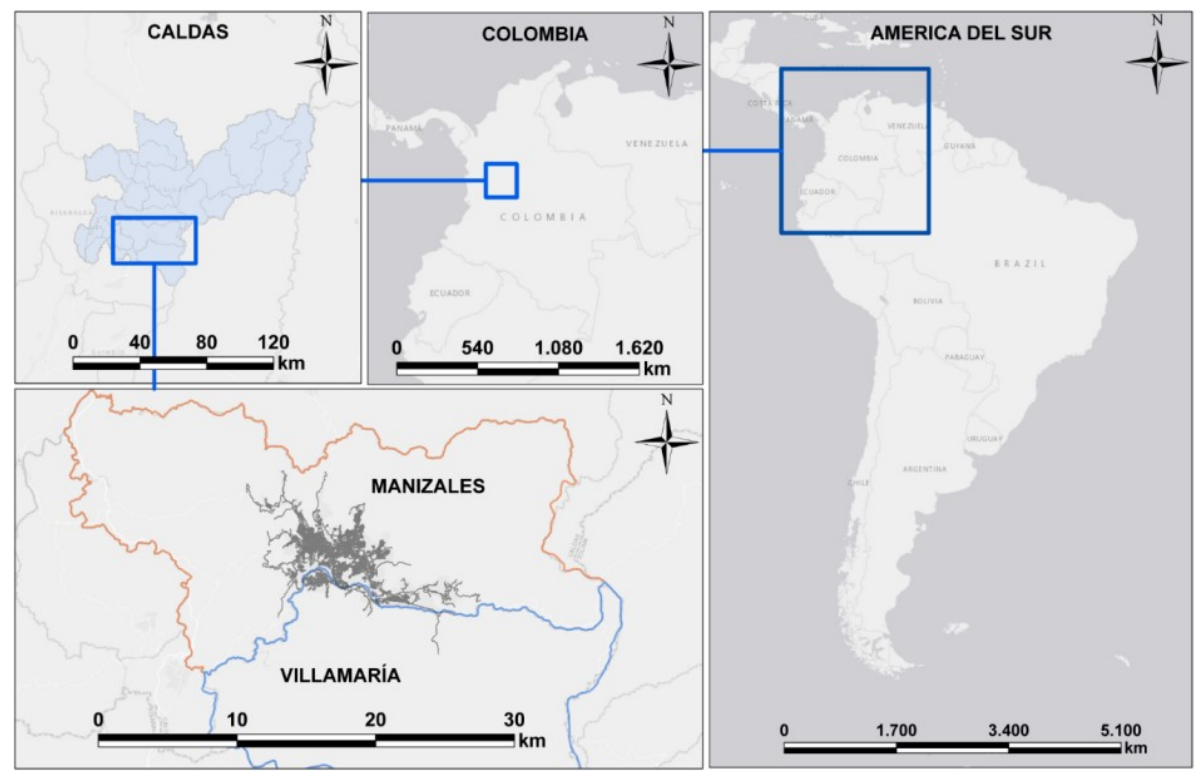

Fig. 1: Localización geográfica del área de estudio, conurbación Manizales - Villamaría.

Se tiene entonces que la precisión del cálculo en las condiciones de accesibilidad se soporta en el detalle de la red de infraestructuras (tipología), así como en la forma como se obtiene dicha variable operativa, en donde actualmente se tienen fuentes de datos proporcionadas por Open Street Map y Google (Weiss et al., 2018), bases de datos que cada día son más precisas; así mismo, es posible utilizar datos recopilados mediante equipos GPS de los viajeros (Carrion y Lenvinson, 2019). Así, considerando los conceptos básicos de accesibilidad, en la presente investigación, se calcula, analiza y comparan las condiciones de accesibilidad media global de la conurbación Manizales - Villamaría en los años 2010 y 2017. Se define el año 2010 como el escenario base para el cálculo de accesibilidad (Escenario 1), con el objetivo de evaluar la evolución en las condiciones de ésta al compararle con la accesibilidad del año 2017 y determinar su nivel de cobertura mediante variables socioeconómicas; ahora bien, entre los años 2010 y 2017 se consideran las intervenciones realizadas por la administración local, proyectos que fueron ejecutados con el fin de mitigar las problemáticas de movilidad identificadas y vividas a lo largo de dicho periodo de tiempo. Las condiciones de accesibilidad en el año 2017 se han calculado a partir de la red de infraestructuras del transporte definida en dos ámbitos diferentes: i) académico e investigativo abordado por el Grupo de Investigación en Movilidad Sostenible de la Universidad Nacional de Colombia (Escenario 2), y ii) un ámbito de consultoría privada contratada directamente por el municipio de Manizales (Escenario 3).

La pregunta de investigación se enmarca en lograr establecer qué tan diferentes son los resultados de las condiciones de accesibilidad media global calculados a partir de una red de infraestructuras del transporte detallada y establecida por investigadores locales (Escenario 2) y otra definida por un equipo consultor externo (Escenario 3) que no vive las condiciones particulares de movilidad de una ciudad y que no ha experimentado la evolución de la misma, según los principales cambios infraestructurales que se han suscitado en un tiempo dado. Ahora bien, es claro que la calidad de vida de los habitantes, en muchos casos, se manifiesta en la facilidad que existe para acceder a las diferentes actividades regulares, inclusive los NAP; es por ello que la evolución de las condiciones de accesibilidad desde el transporte, es un factor que debe medirse con la mayor precisión posible, encontrando que se considera de suma importancia el demostrar la existencia de importantes diferencias que existen al analizar la ciudad a partir de una u otra red de infraestructura, pues de allí se desprenden una serie de decisiones coyunturales, que afectarían profundamente las características de accesibilidad y movilidad en la ciudad; además de ello, las repercusiones que se tendrían en tomar datos como ciertos, pueden afectar o beneficiar a diferentes actores involucrados en el diario vivir de una población, comunidad y/o región, dado que los esfuerzos en términos de una política pública, podrían estar erróneamente enfocados o direccionados al no tenerse resultados que correspondan a las condiciones actuales de infraestructura del transporte, debiéndose evitar precisiones aparentes (Graser y Dragaschnig, 2020). Posterior a la breve introducción, se presenta la metodología de investigación, para después llegar a los resultados y discusión, y finalizar con las conclusiones más relevantes del presente trabajo investigativo. 


\section{METODOLOGÍA}

La metodología de investigación abordada comprende el desarrollo de seis fases (Figura 2) principales de carácter consecutivo, las cuales sintetizan los procesos de análisis y se describen a continuación.

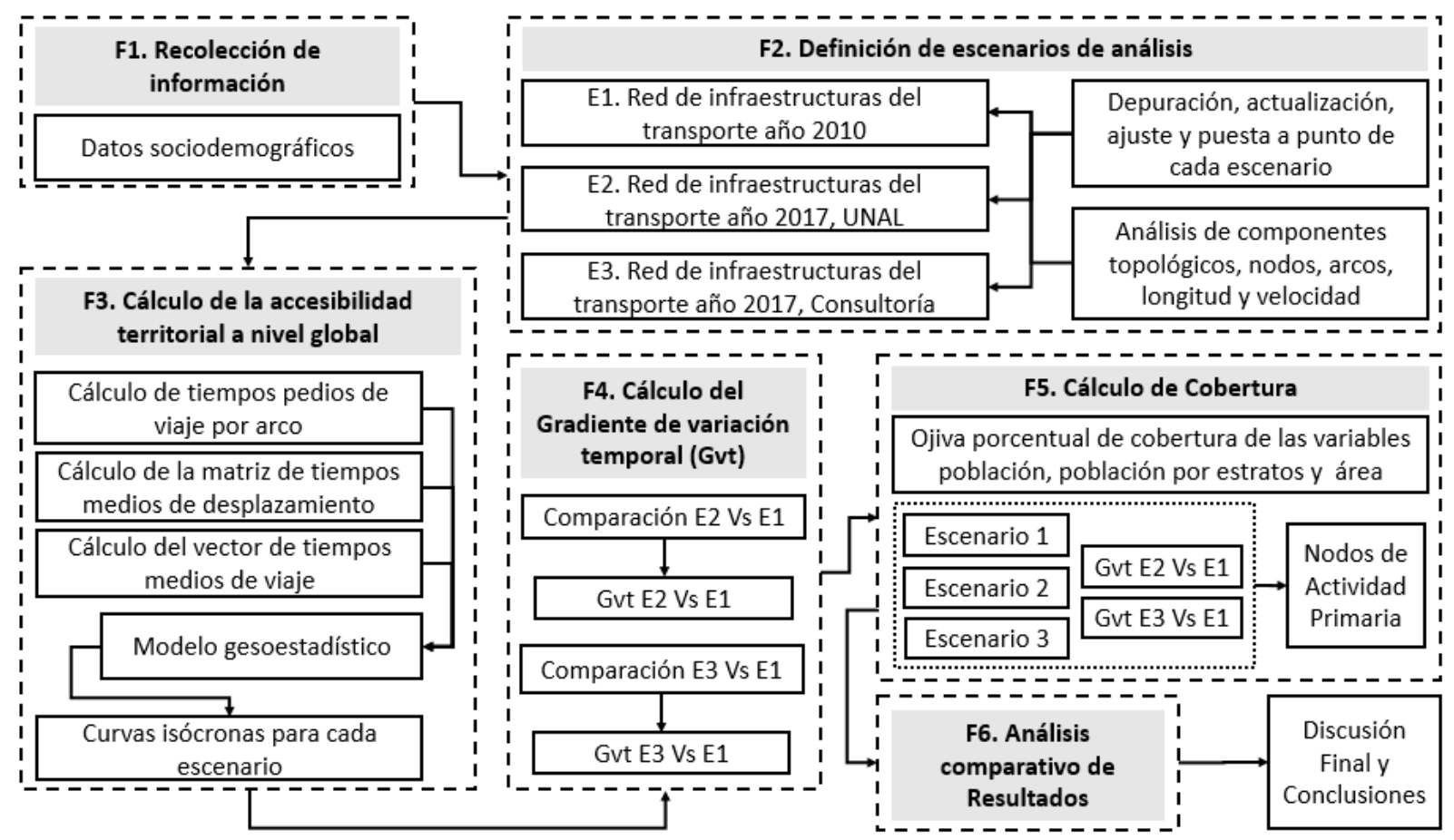

Fig. 2: Metodología de investigación abordada.

Fase 1. Recolección de información. Como información secundaria se tiene toda aquella relacionada con datos sociodemográficos del área conurbada Manizales - Villamaría, como son el polígono de barrios, número de habitantes, estrato socioeconómico, área, etc. Así mismo, se revisa y ajusta toda la información que será cargada en las capas de información, con el fin de realizar análisis de superposición con las curvas de tiempo medio que se obtengan en una fase posterior. Los datos operativos de velocidad hacen relación al promedio de velocidad de todos los modos de transporte que usan la infraestructura.

Fase 2. Definición de escenarios de análisis: En esta fase de la investigación se depuran, actualizan, ajustan y ponen a punto las tres redes de trabajo, las cuales son las que permitirán establecer los escenarios a estudiar. Para evaluar las condiciones de accesibilidad entre el año 2010 y 2017 se procede al refinamiento de cada una de las redes de infraestructuras del transporte para cada uno de los 3 escenarios analizados: i) El escenario 1 comprende el estado y funcionamiento de la red vial para el año 2010, la cual toma como referencia los componentes establecidos en el plan maestro de movilidad de la ciudad a la fecha; por lo tanto, se procede a realizar el refinamiento de la red de transporte sin incluir modificaciones adicionales; ii) el escenario 2 es base para el cálculo de la accesibilidad media global a partir de la red de trabajo ajustada y validada por el equipo de investigación en Movilidad Sostenible de la Universidad Nacional de Colombia sede Manizales, esta red incluye todas las intervenciones infraestructurales ejecutadas por la administración municipal entre los años 2010 y 2017; y iii) el escenario 3 corresponde al cálculo de la accesibilidad media global de la ciudad basada en la red de infraestructuras del transporte con la que trabajó la consultoría contratada por la administración municipal en el año 2017. Las redes deben cumplir con la teoría de grafos, evaluando su desempeño a través de sus principales componentes topológicos, nodos (intersecciones viales), arcos (vías) (Weber, 2016)., longitud, velocidad (Liu \& Zhu, 2004).

Fase 3. Calculo de accesibilidad territorial a nivel global: Una vez establecidos los tres escenarios, se procede a realizar el cálculo de las curvas isócronas de accesibilidad a partir del tiempo medio de viaje entre cada par de nodos, considerando el costo de desplazamiento sobre cada arco mediante la relación de velocidad y longitud como se muestra en la Ecuación (1); donde: i es el arco en cuestión, Tvi es el tiempo medio de viaje para el arco i; $L_{i}$ es la longitud del arco i y $V_{i}$ es la velocidad del arco i,

$$
T v_{i}=\frac{L_{i}}{V_{i}} \quad i=1,2,3, \ldots, n
$$


Luego de obtenerse el costo de desplazamiento sobre cada arco, se ensambla la matriz de tiempos de desplazamiento, en la cual se define el camino mínimo desde un nodo de origen i y uno de destino j (algoritmo Dkjistra), para posteriormente determinar el tiempo de viaje promedio de cada nodo mediante la Ecuación (2) (Perilla et al., 2018); donde: $\mathrm{T}_{\mathrm{vi}}$ es el tiempo de desplazamiento promedio del punto $\mathrm{i}$; $\mathrm{tv}_{\mathrm{ij}}$ el tiempo de desplazamiento del punto i al punto j y $\mathrm{n}$, el número total de puntos analizados, debiéndose tener en cuenta las correspondientes penalidades por giro de la red en estudio (Cardona, 2018); es decir, este cálculo se relaciona con el promedio de los tiempos de viaje entre todos los nodos de la red vial (Moncada et al., 2018).

$$
\overline{T_{v l j}}=\frac{\sum_{i=1}^{n} t_{v i j}}{(n-1)} \quad i=1,2,3, \ldots, n ; j=1,2,3, \ldots, \mathrm{n}
$$

Finalmente, se relacionan los tiempos de desplazamiento con las coordenadas geográficas de cada nodo y mediante modelos geoestadísticos, se construyen las curvas isócronas. El método geoestadístico utilizado es el de Kriging ordinario con semivariograma lineal; modelo mediante el cual se han obtenido aproximaciones más acertadas y menores errores cuando la distancia entre puntos a interpolar es corta (Li y Heap, 2008), en donde el semivariograma caracteriza las propiedades de dependencia entre nodos de un mismo campo, observado por medio de la Ecuación (3) (Perilla et al., 2018); donde: $Z_{x}$ es el valor de la variable para un nodo con coordenadas $\mathrm{x} ; \mathrm{Z}_{\mathrm{x}+\mathrm{h}}$ el valor muestral siguiente apartados por una distancia $\mathrm{h}$ y $\mathrm{n}$ el número de parejas apartados por la distancia $h$.

$$
\overline{\gamma_{(h)}}=\frac{\sum\left(Z_{(x+h)}-Z_{(x)}\right)^{2}}{2 n}
$$

Fase 4. Cálculo del Gradiente de variación temporal (Gvt). Este cálculo permite comparar las condiciones de accesibilidad territorial a nivel global entre dos períodos de tiempo; es decir, a partir de los vectores de tiempo mínimo de viaje de cada escenario, se realiza una comparación con el fin de conocer que tan diferentes son los tiempos entre escenarios y observar en qué lugares de la ciudad hubo mayor o menor impacto. La Ecuación 4 presenta la expresión matemática mediante la cual se realiza el respectivo cálculo, en donde TvE1 es el vector de tiempos mínimos de viaje para el escenario1, TvEj es el vector de tiempo mínimo de viaje para cada los escenarios 2 y 3 , respectivamente, lo cual permite obtener un nuevo vector, el vector gradiente de variación temporal, con el que se procede a ejecutar el modelo geoestadístico descrito en la fase 3 , con el fin de obtener las curvas de variación porcentual entre escenarios.

$$
G v t=\frac{T_{v E 1}-T_{v E j}}{T_{v E 1}} * 100, \text { donde } \mathrm{j}=2 \text { y } 3 \text { para cada escenario correspondiente al año } 2017
$$

Fase 5. Calculo de cobertura. En esta fase se relacionan las variables sociodemográficas, área, población, estratificación socioeconómica y cantidad de Nodos de Actividad Primaria (NAP), con las curvas isócronas de accesibilidad territorial a nivel global obtenidas para cada uno de los escenarios y para los dos gradientes de variación temporal. Una vez seleccionadas las variables a relacionar, se cruza la información mediante la extensión "Geoprocessing" de ArcMap; posteriormente se realizó el manejo de los datos por medio de Microsoft Excel y se construyeron las correspondientes ojivas de cobertura porcentual. Los estratos son una clasificación de la población según su condición socioeconómica de una manera jerarquizada; para el caso de Manizales, se cuenta con un total de 6 estratos socioeconómicos, siendo 1 el de menor capacidad económica y 6 el de mayor capacidad.

Fase 6. Análisis comparativo de resultados. En esta fase final se comparan los resultados tanto desde el punto de vista gráfico, como desde el punto de vista cuantitativo, en relación con la variación en los porcentajes de cobertura para cada escenario y para cada gradiente de variación temporal. Esta comparación permitirá soportar la discusión y conclusiones de la investigación.

\section{RESULTADOS Y DISCUSIÓN}

En la Tabal 1 se presentan las principales diferencias encontradas entre las tres redes de infraestructura de estudiadas, el escenario 1 (2010) y los mencionados escenarios 2 y 3, ambos definidos para el año 2017 , pero en diferentes ámbitos, uno académico e investigativo (Escenario 2) y el otro, un ámbito de consultoría privada (Escenario 3). Analizando la longitud definida para cada una de las redes, se observa que la correspondiente al escenario 2 (2017) es 4,6\% mayor que la establecida en el escenario 1 (2010), situación que se comprende lógica, encontrando que, según el tipo de vía, para la red del escenario 1 no se tenían los tipos de vía nacional y departamental; no obstante, al comparar el escenario 1 (2010) con el escenario 3 (2017), este último es un $46,6 \%$ menor, dando a entender que la red de infraestructuras del transporte se redujo para el año 2017 en aproximadamente 299,9 Kilómetros, registrando la mayor reducción en los tipos de vías locales, principales, secundaria y colectora, respectivamente. Ahora bien, en relación con el número 
de nodos que soportan estructuralmente le red, el escenario 1 refiere un total de 7540 nodos, el escenario 2 un total de 8163 nodos y el escenario 3 un total de 1815 nodos; lo anterior indica que el escenario 3 representa menos del $25 \%$ de los nodos que se encontraban identificados inicialmente para el escenario 1 , mientras que para el escenario 2, el número de nodos aumentó, en relación con el escenario 1 en un $8,3 \%$, lo cual es más lógico, al comprender que entre ambos escenarios existen siete años de diferencia, período de tiempo durante el cual se espera que la red de infraestructura de una ciudad aumente y que la información de éstas redes sea cada vez más detallada y no se simplifique de una forma tan generalizada como la utilizada en el escenario 3 , situación que también se comprueba al analizar el número de arcos que componen cada uno de los escenarios, 10290 arcos, 10784 arcos y 5308 arcos para cada escenario respectivamente.

Tabla 1. Comparación de las características de las redes de infraestructura de los escenarios 1 (2010), 2 y 3 (2017).

\begin{tabular}{|c|c|c|c|c|c|c|c|c|c|c|c|c|c|c|c|}
\hline \multirow[b]{2}{*}{$\begin{array}{l}\frac{\pi}{2} \\
\frac{1}{0} \\
0 \\
\stackrel{0}{0}\end{array}$} & \multicolumn{5}{|c|}{ Escenario 1 (2010) } & \multicolumn{5}{|c|}{ Escenario 2 (2017) } & \multicolumn{5}{|c|}{ Escenario 3 (2017) } \\
\hline & 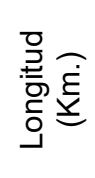 & 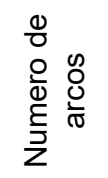 & 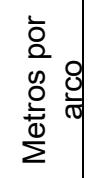 & 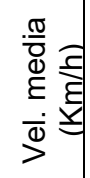 & 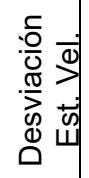 & 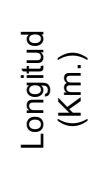 & 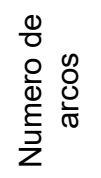 & 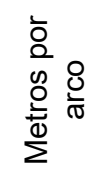 & 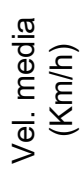 & 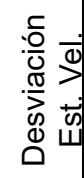 & 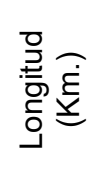 & 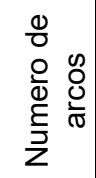 & 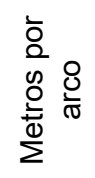 & 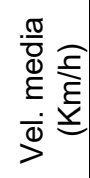 & 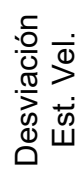 \\
\hline r pal. & 65,4 & & , & | & 10,6 & 65,4 & & & & 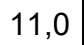 & U, & 140 & 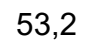 & 20,0 & 13,0 \\
\hline Sec. & 60,1 & 977 & 61,5 & 21,7 & 9,5 & 60,1 & 1004 & 59,8 & 22,1 & 9,7 & 48,8 & 1028 & 47,5 & 18,6 & 8,8 \\
\hline Col. & 100,4 & 1000 & 53,2 & 18,4 & 7,9 & 100,4 & 1982 & , & 10,2 & $T, I$ & 84,6 & 1366 & U, & 10,7 & 9,1 \\
\hline Loc. & 376,4 & 5901 & 63,8 & 14,4 & 7,7 & 358,6 & 5950 & 60,3 & 13,9 & 6,8 & 136,2 & 2054 & 6,3 & 14,8 & 7,4 \\
\hline Nac. & NE & NE & F & NE & NE & 26,5 & 5 & 10 & 3 & 11 & $118 \mathrm{~s}$ & 40 & 370,9 & 37 & 14,0 \\
\hline Dep. & NE & NE & NE & NE & $\mathrm{NE}$ & 18,4 & 140 & 131,3 & 30,5 & 10,3 & 19,9 & 72 & 275,8 & 20,9 & 10,2 \\
\hline Semip. & 41,8 & 070 & 61,7 & 12,1 & 7,0 & 44,5 & 735 & $0, v$ & 12,1 & 6,7 & TVL & $\mathrm{NE}$ & Nve & NE & NE \\
\hline Total & 44,0 & 290 & 62,6 & 19,7 & 8,5 & 673,9 & 10784 & 62,5 & 23,1 & 9,0 & 344,1 & 5308 & 64,8 & 22,7 & 10,4 \\
\hline
\end{tabular}

Por otra parte, en la variable metros por arco, se observa que los tres escenarios poseen un número de metros similar; no obstante, al analizar los metros por arco según el tipo de vía, se encuentran algunas diferencias al respecto, lo cual refuerza nuevamente la hipótesis de que el escenario 3 realiza una generalización muy fuerte de la red de infraestructuras real de la ciudad. En relación con la velocidad media de operación, se encuentra que los valores de los escenarios 2 y 3 son bastante consistentes con $23,1 \mathrm{Km} / \mathrm{h}$ y $22,7 \mathrm{Km} / \mathrm{h}$, indicando que dicha variable aumento al relacionarse con el valor del escenario 1, 19,7 Km/h; así mismo, se encuentra que, según el tipo de vía, el valor de la desviación estándar es mayor en todos los casos para el escenario 3 , excepto en las vías secundarias. El tener una red de escenario 3 tan generalizada, hace que los resultados de un estudio de accesibilidad no posean la precisión necesaria, a pesar que los datos de características operativas sobre la red vial, vengan de fuentes de información adecuadas y cumplan con un número estadísticamente significativo en términos del tamaño de la muestra. El problema radica precisamente en que, al generalizar la red, se dejan de trazar vías, por ejemplo, dobles calzadas, que para un análisis de accesibilidad es necesario tener claramente separadas, pues la velocidad operativa en un sentido seguramente varía en relación con el otro sentido, situación que no tiene en cuenta el escenario definido y propuesto por la consultoría privada.

Ahora bien, los resultados del estudio de las condiciones de accesibilidad media global, para los tres escenarios, se presenta en tres formatos: i) el primero es el mapa de curvas de accesibilidad de la ciudad, en el cual se identifican los tiempos promedio de desplazamiento entre los distintos puntos en intervalos de 5 minutos, ii) el segundo formato son las ojivas porcentuales de cobertura acumulada según estrato socioeconómico, en éste se identifican cuáles son los estratos que presentan mejor cobertura respecto a un tiempo medio de viaje en particular, y iii) tercero son las ojivas porcentuales de cobertura acumulada según las variables población y área. Posteriormente, con el fin de detallar un poco más las diferencias entre el uso de una red de infraestructuras detallada (escenario 2) y otra generalizada (escenario 3 ), se realiza un análisis comparativo de la cobertura de los Nodos de Actividad Primaria, tanto para dichos escenarios como para los resultados obtenidos relacionados con el Gradiente de variación temporal. En la Figura 3 se presenta el mapa de las curvas isócronas de tiempo medio de viaje para el escenario 1 (año 2010), se obtuvo que el valor de la menor curva isócrona es de 25 minutos, localizado a lo largo del corredor de movilidad que une el Central Bussines District (CBD) y el denominado Sector del Cable en sentido oriente - occidente y viceversa. Las características de movilidad para dicho año garantizaban un mejor desplazamiento hacia los nodos localizados alrededor del CBD de la ciudad, dejando a un lado zonas residenciales periféricas como son La Linda (al noroccidente) y el conjunto La Enea - Maltería (al suroriente), los cuales necesitaban de hasta 70 minutos de tiempo medio de viaje para llegar a ellos. 


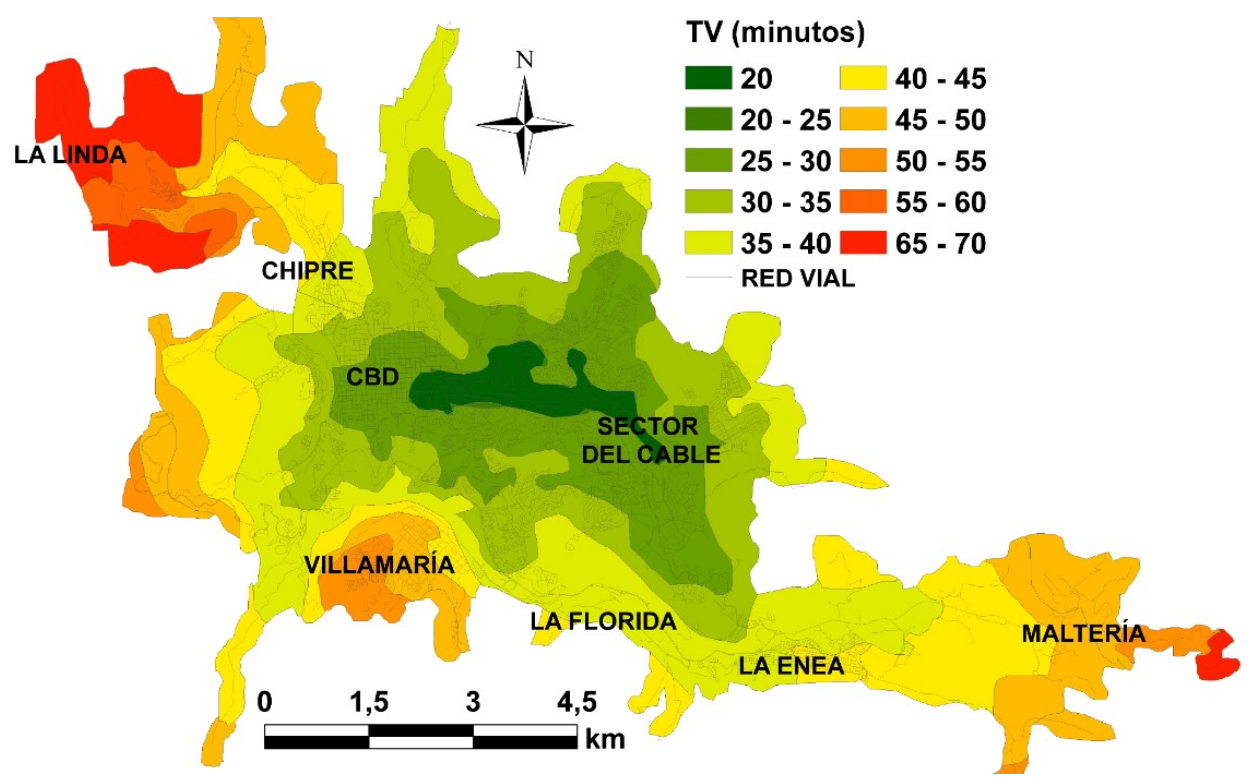

Fig. 3: Curvas de accesibilidad territorial a nivel global para el escenario 1 (año 2010).

La Figura 4a presenta la variación de cobertura porcentual acumulada para las variables población y área, encontrando que para un $50 \%$ de cobertura, se requiere de un tiempo de viaje de entre 26 y 32,8 minutos, respectivamente. El nivel de cobertura acumulada por estrato socioeconómico se presenta en la Figura 4b, se tiene que, el estrato 5 cubre la totalidad de su población con tiempos de viaje de hasta 30 minutos, seguido de los estratos $2,1,6,4$ y 3 , con $87,8 \%, 81,0 \%, 78,8 \%, 73,9 \%$ y $62,3 \%$, respectivamente. Por otro lado, los estratos 1 y 3 presentan las coberturas más desfavorables, en el primer caso para tiempos de viaje menores a 27 minutos y en el segundo caso para tiempos de viaje mayores a 27 minutos. Para una cobertura del $50 \%$ de la población de cada estrato, los tiempos de viaje varían de 22,8 minutos para el estrato 5 hasta 26,9 minutos para los estratos 1 y 3 . Realizando un cálculo del tiempo de viaje necesario por estrato, ponderado por la población de cada uno de estos, se tiene que las personas del estrato 5 son las que menor tiempo medio de viaje refieren, llegando a 25,8 minutos, seguido del estrato 4 con 28,8 minutos, el estrato 6 con 28,9 minutos, estrato 2 con 29,1 minutos, estrato 1 con 30,0 minutos y finalmente el estrato 3 con 31,7 minutos.
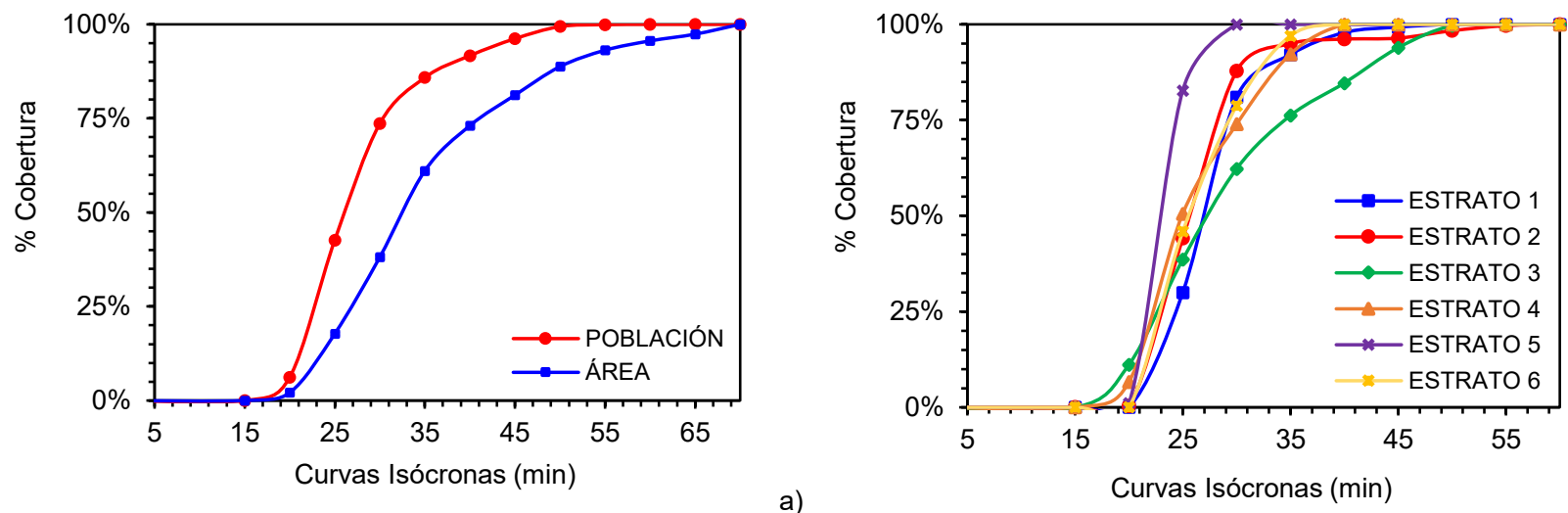

b)

Fig. 4: Ojivas porcentuales de cobertura acumulada en el escenario 1, a) según las variables población y área; b) según estrato socioeconómico.

En la Figura 5 se presenta el cambio en el tiempo de viaje para el caso del escenario 2; la curva isócrona de menor valor es de 20 minutos, curva que comparada con la obtenida en el escenario 1, se observa cómo se extiende en sentido occidente-oriente a lo largo de su principal eje de movilidad, así mismo, se extiende en sentido norte-sur. Las zonas con mayores tiempos de viaje siguen ubicadas en la periferia de la ciudad, sin embargo, ahora son unas menores áreas ubicadas en el sector noroccidental de la ciudad (La Linda). La Figura 6a presenta la variación de cobertura porcentual acumulada para las variables población y área, encontrando que para un $50 \%$ de cobertura, se requiere de un tiempo de viaje de entre 21 y 24,8 minutos, respectivamente. Por otra parte, el nivel de cobertura acumulada por estrato socioeconómico se presenta en la Figura $6 \mathrm{~b}$, encontrándose que, el estrato 5 cubre la totalidad de su población con tiempos de viaje de hasta 26 minutos, seguido de los estratos $6,1,4,2$ y 3 . Para una cobertura del $50 \%$ de la población de cada estrato, los tiempos de viaje varían de 18,2 minutos para el estrato 5 hasta 21,8 minutos para el estrato 1 . Realizando un cálculo del tiempo de viaje necesario por estrato, ponderado por la población de cada uno de estos, se 
tiene que las personas del estrato 5 son las que menor tiempo medio de viaje refieren, llegando a 20,8 minutos, seguido del estrato 6 con 22,6 minutos, el estrato 4 con 23,5 minutos, estrato 1 con 24,1 minutos, estrato 3 con 24,2 minutos y finalmente el estrato 2 con 24,4 minutos.

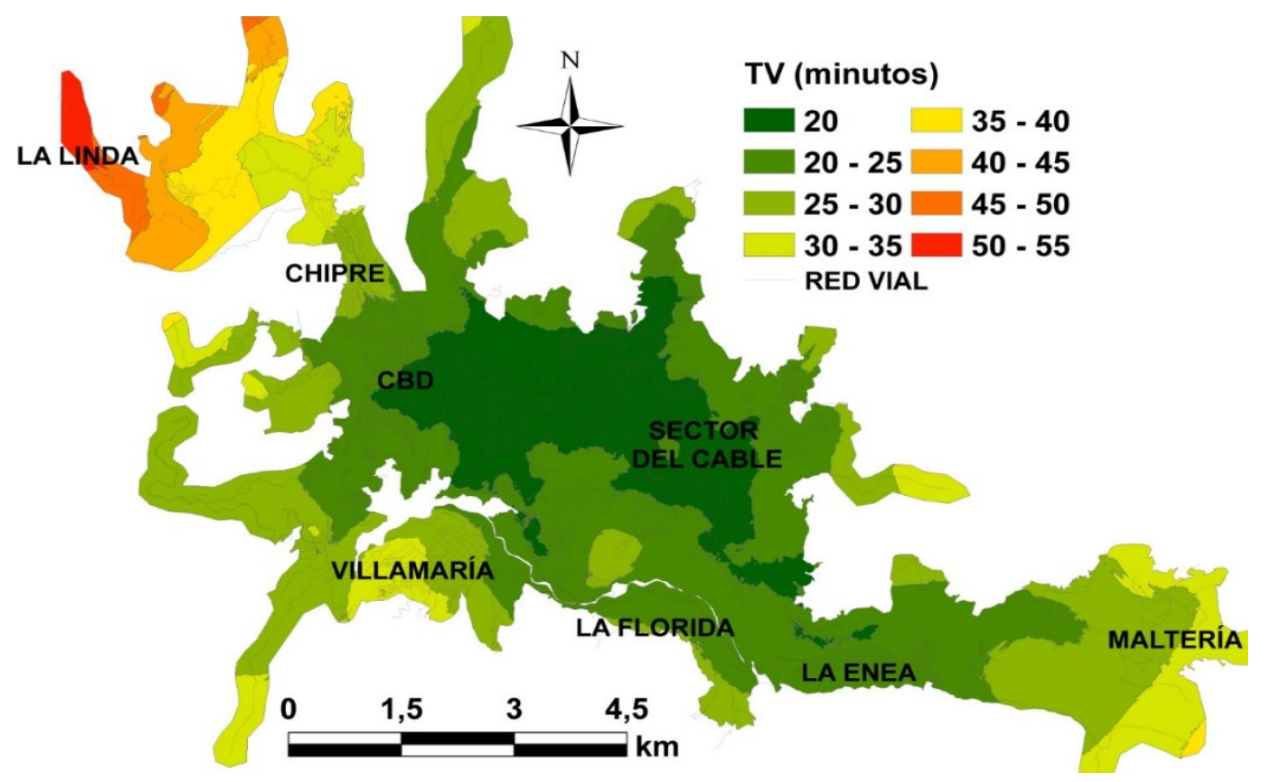

Fig. 5: Curvas de accesibilidad territorial a nivel global para el escenario 2 (año 2017).
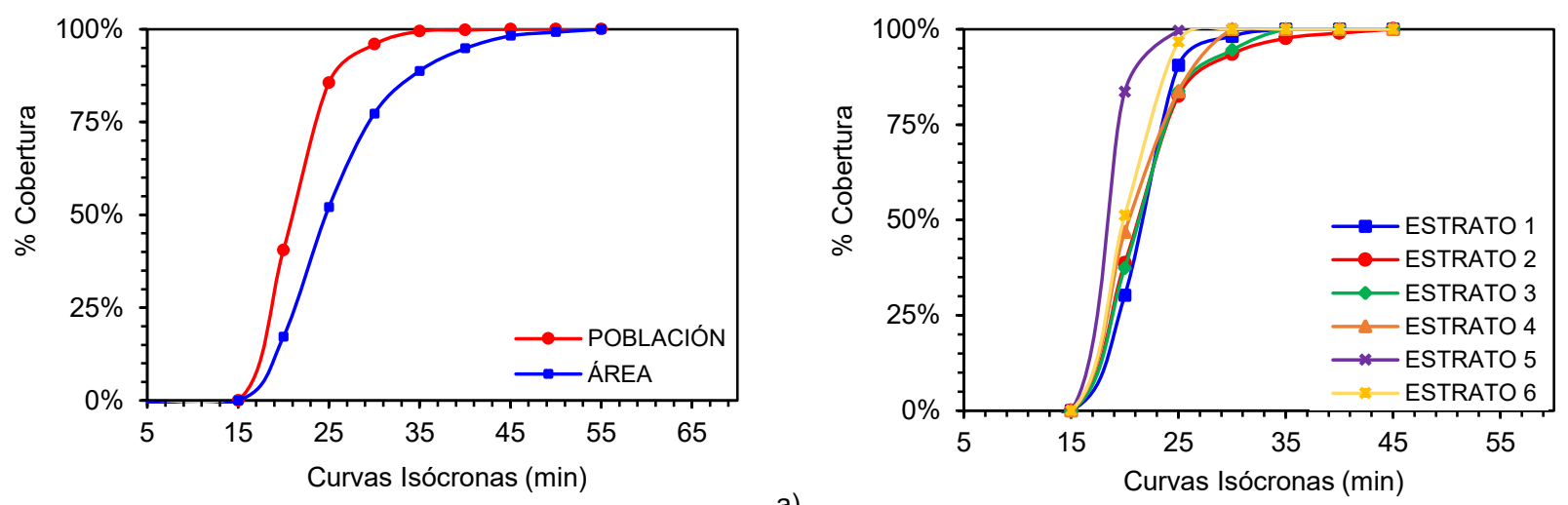

a)

Fig. 6: Ojivas porcentuales de cobertura acumulada en el escenario 2, a) según las variables población y área; b) según estrato socioeconómico.

En la Figura 7 se presentan los tiempos medios de viaje obtenidos para el escenario 3 , los cuales se encuentran en el rango de 8 a 40 minutos, los cuales se reducen a más de la mitad en comparación con los escenarios 1 y 2. La Figura 8a presenta la variación de cobertura porcentual acumulada para las variables población y área, encontrando que para un $50 \%$ de cobertura, se requiere de un tiempo de viaje de entre 14,5 y 19 minutos, respectivamente. Así mismo, el nivel de cobertura acumulada por estrato socioeconómico se presenta en la Figura 8b, encontrándose que, el estrato 5 cubre la totalidad de su población con tiempos de viaje de hasta 20 minutos, seguido de los estratos 4, 6, 3, 1 y 2. Para una cobertura del $50 \%$ de la población de cada estrato, los tiempos de viaje varían de 12,9 minutos para el estrato 5 hasta 15,8 minutos para el estrato 6. Realizando un cálculo del tiempo de viaje necesario por estrato, ponderado por la población de cada uno de estos, se tiene que las personas del estrato 5 son las que menor tiempo medio de viaje refieren, llegando a 15,9 minutos, seguido del estrato 4 con 16,3 minutos, el estrato 3 con 17,8 minutos, estrato 1 con 18,0 minutos, estrato 6 con 18,1 minutos y finalmente el estrato 2 con 18,6 minutos.

Ahora bien, con el fin de comparar los resultados de cobertura obtenidos en cada escenario, en la Tabla 2 se presentan los tiempos medios de viaje ponderados por población para cada uno de los estratos, y la relación de los escenarios 2 y 3 con el escenario 1. 


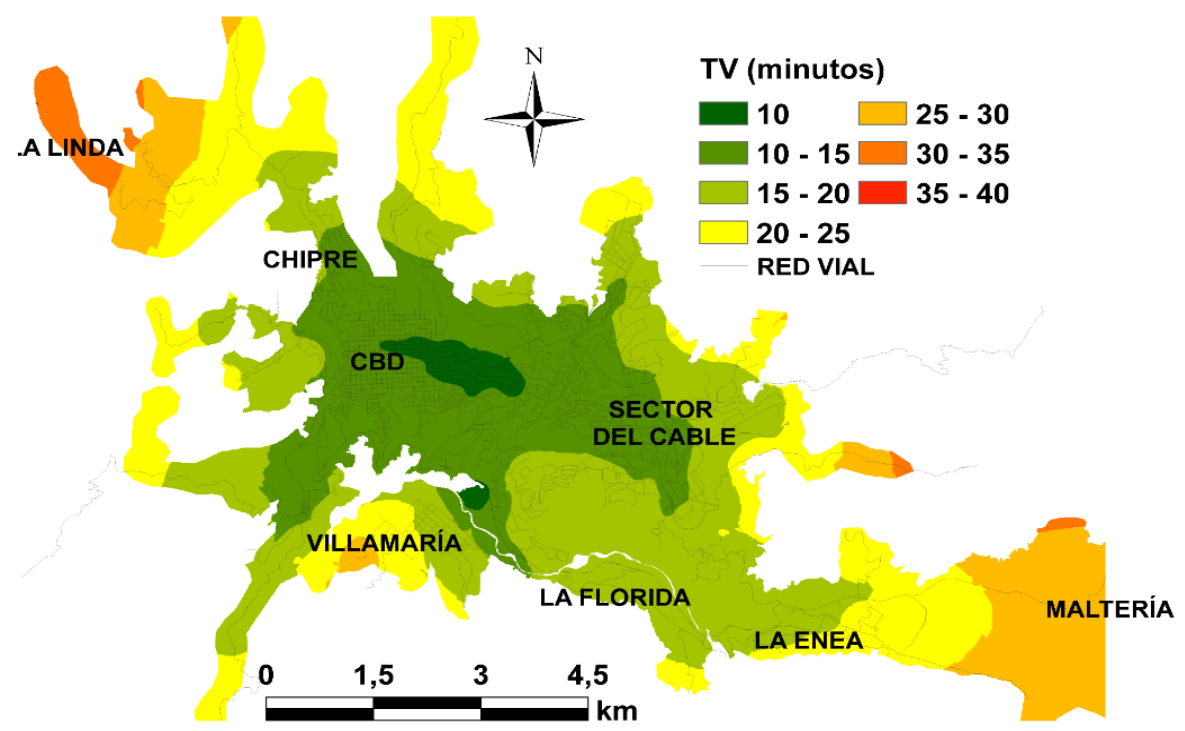

Fig. 7: Curvas de accesibilidad territorial a nivel global para el escenario 3 (año 2017).
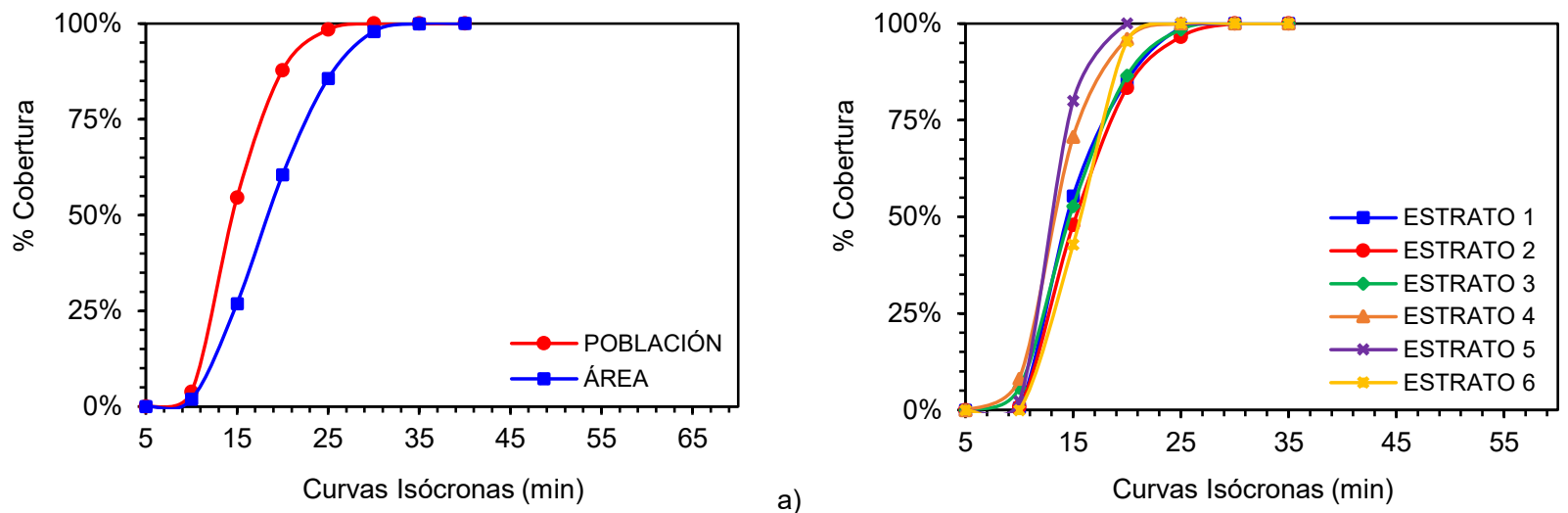

Fig. 8: Ojivas porcentuales de cobertura acumulada en el escenario 3, a) según las variables población y área; b) según estrato socioeconómico.

Tabla 2. Comparación de los tres escenarios estudiados tiempo medio de viaje ponderado por población.

\begin{tabular}{|c|c|c|c|c|c|}
\hline \multirow{2}{*}{ Estrato } & \multicolumn{5}{|c|}{ Tiempo medio de viaje (minutos) ponderado por población } \\
\cline { 2 - 6 } & Escenario_1 & Escenario_2 & Escenario_3 & E_1 Vs E_2 & E_1 Vs E_3 \\
\hline 1 & 30,0 & 24,1 & 18,0 & $19,8 \%$ & $40,0 \%$ \\
\hline 2 & 29,1 & 24,4 & 18,6 & $16,1 \%$ & $36,2 \%$ \\
\hline 3 & 31,7 & 24,2 & 17,8 & $23,5 \%$ & $43,7 \%$ \\
\hline 4 & 28,8 & 23,5 & 16,3 & $18,6 \%$ & $43,6 \%$ \\
\hline 5 & 25,8 & 20,8 & 15,9 & $19,2 \%$ & $38,4 \%$ \\
\hline 6 & 28,9 & 22,6 & 18,1 & $21,8 \%$ & $37,4 \%$ \\
\hline Total & 30,2 & 23,9 & 17,8 & $20,8 \%$ & $41,2 \%$ \\
\hline
\end{tabular}

Analizando el tiempo medio de viaje ponderado por población, de forma general, se tiene que en el año 2010 (Escenario 1), dicho valor era levemente superior a la media hora, mientras que para el escenario 2 se redujo en un 20,8\%, llegando a un tiempo de 23,9 minutos; no obstante, al comprar el tiempo del escenario 1 con el tiempo del escenario 3, se observa una reducción del mismo superior al 40\%, llegando a 17,8 minutos, valor demasiado bajo teniendo en cuenta las características de la ciudad y lo que se vive en términos de movilidad; es decir, es posible que se hayan reducido los tiempos medios de viaje, dadas las nuevas infraestructuras, pero es poco probable que dicha reducción sea tan alta como la expresada por el escenario 3. Por otra parte, el estrato con menor tiempo de viaje ponderado por población, para los tres escenarios es el estrato cinco; el estrato con mayor tiempo medio de viaje en el escenario 1 es el estrato tres, mientras que para los escenarios 2 y 3 es el estrato dos. Por otra parte, se encontró que el estrato que refiere mayor variación porcentual en la 
reducción del tiempo medio de viaje ponderado por población, es el estrato tres, con valores de reducción del $23,5 \%$ y $43,7 \%$ para los escenarios 2 y 3 , respectivamente.

Al realizar los cálculos del gradiente de variación temporal entre el escenario 1 y el escenario 2 , se obtienen las curvas de isoahorro porcentual que se presentan en la figura 9; se observa que el sector que refiere un menor porcentaje de ahorro entre ambos escenarios es el noroccidente (La Linda), llegando a valores de ahorro de hasta el $20 \%$, no obstante, en este mismo sector se encuentran zonas que refieren ahorros de hasta el $65 \%$, zonas que se relacionan con los accesos a veredas o fincas que se encontraban desconectadas de la red de transporte en el escenario 1 y que para el escenario 2 ya referían algún nivel de conexión; así mismo, otros sectores que refieren el mayor porcentaje de ahorro porcentual se ubican al sur, en los sectores de La Florida y Villamaría, sectores estos que en conjunto con La Enea y Maltería (Oriente), son los de mayor beneficio en términos de ahorro de tiempo al comparar éstos dos escenarios, situación bastante consecuente con el desarrollo de las obras de conexión occidente-oriente y viceversa al sur del área de estudio.

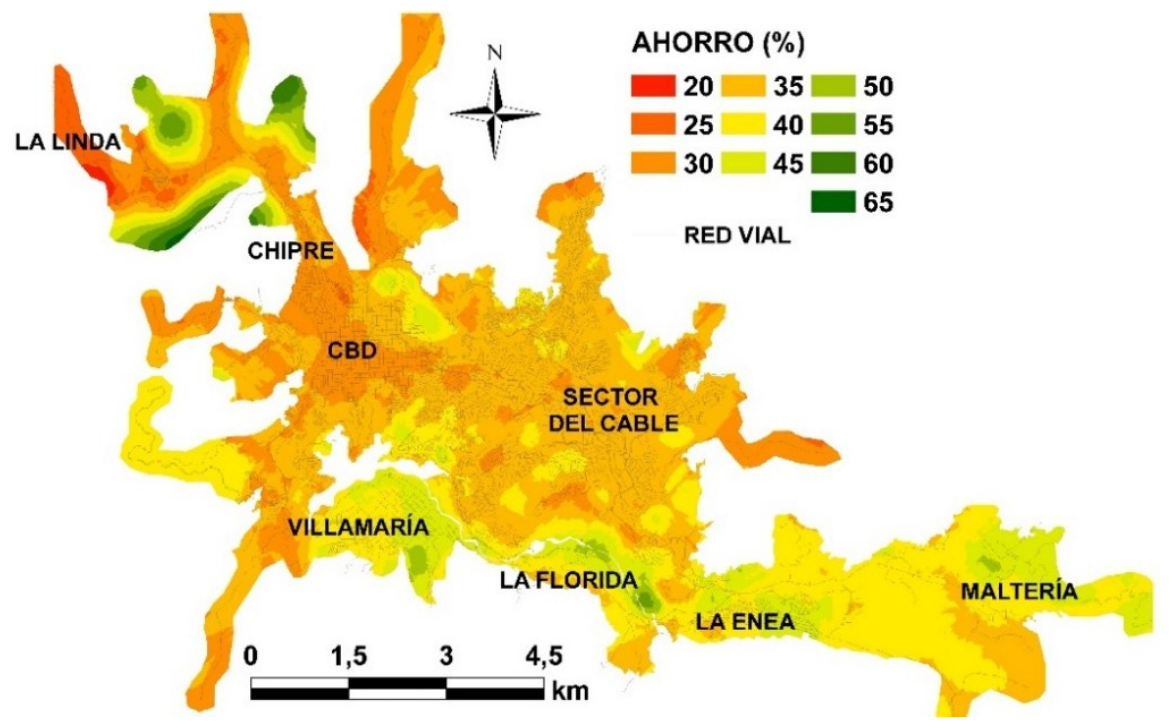

Fig. 9: Gradiente de variación temporal entre escenario 1 y escenario 2.

En la figura 10a se presenta la variación de la cobertura de las curvas de isoahorro porcentual para las variables población y área, se observa que para un $50 \%$ de cobertura se tienen ahorros porcentuales de $32,5 \%$ y $33,5 \%$, respectivamente, al comparar el escenario 1 y el escenario 2 . Por otra parte, en la figura $10 \mathrm{~b}$ se observa la relación entre las curvas de isoahorro porcentual y la cobertura poblacional según estrato, se observa, que para un $50 \%$ de cobertura poblacional, el estrato que mayor ahorro porcentual refiere es el estrato 1 con un $33,9 \%$, seguido en su orden por el estrato $6(33,6 \%)$, estrato $2(33,1 \%)$, estrato $5(32,3 \%)$ y estrato $4(31 \%)$, orden porcentual diferente en relación con la variación del tiempo medio de viaje ponderado por población, como se observó con anterioridad.
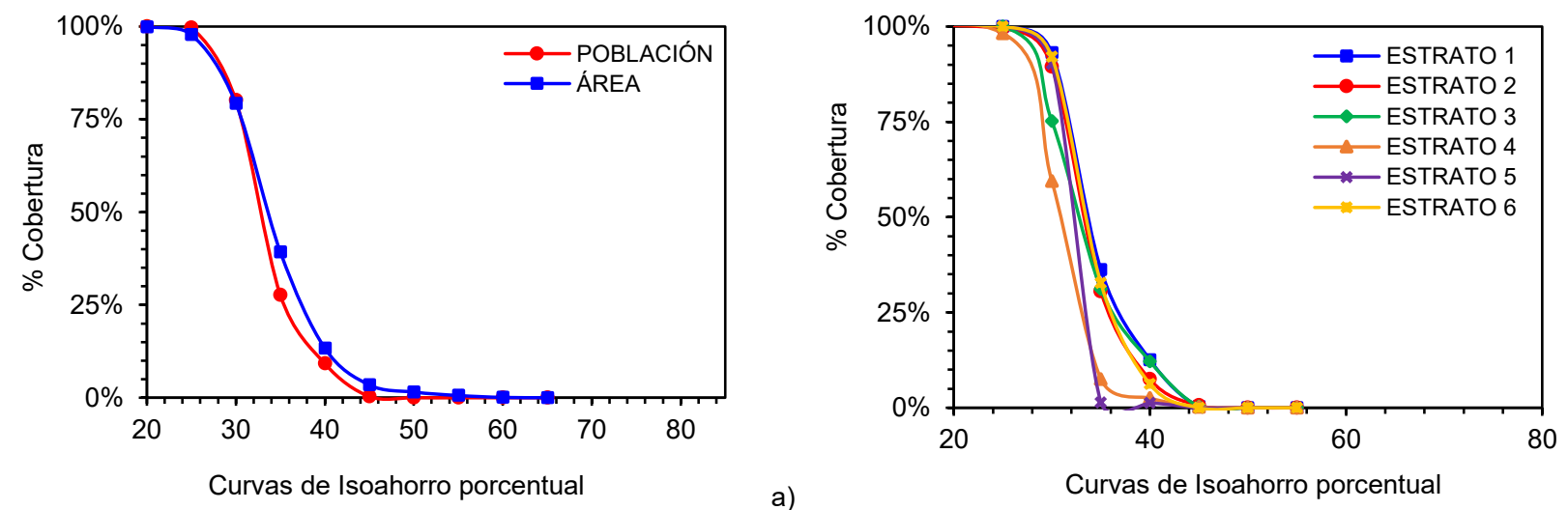

Fig. 10: Ojivas porcentuales de cobertura acumulada en el escenario 3, a) según estrato socioeconómico; b) según las variables población y área.

Ahora bien, al realizar los cálculos del gradiente de variación temporal entre el escenario 1 y el escenario 3 , se obtienen las curvas de isoahorro porcentual que se presentan en la figura 11; en este caso se obtienen resultados diametralmente opuestos a los obtenidos al comparar el escenario 1 y el escenario 2. Por ejemplo, se observa que el sector que refiere un menor porcentaje de ahorro entre ambos escenarios es el oriente 
(Maltería, La Enea), llegando a valores de ahorro entre el $30 \%$ y el $45 \%$; por otra parte, el sector que refiere un mayor porcentaje de ahorro se ubican en el sector occidente, situación contraria a la identificada con al comparar los escenarios 1 y 2, como se presentó con anterioridad. En la figura 12a se presenta la variación de la cobertura de las curvas de isoahorro porcentual para las variables población y área, se observa que para un $50 \%$ de cobertura se tienen ahorros porcentuales de $55,4 \%$ y $52 \%$, respectivamente, al comparar el escenario 1 y el escenario 3 , valores estos muy superiores al compararlos con los obtenidos entre el escenario $1 \mathrm{y}$ el escenario 2. Por otra parte, en la figura $12 \mathrm{~b}$ se observa la relación entre las curvas de isoahorro porcentual y la cobertura poblacional según estrato; se observa, que para un $50 \%$ de cobertura poblacional, el estrato que mayor ahorro porcentual refiere es el estrato 4 con un $57,9 \%$, seguido en su orden por los estratos 1 y $3(55,8 \%)$, estratos 2 y $5(52 \%)$ y estrato $6(48,5 \%)$, orden porcentual diferente en relación con la variación del tiempo medio de viaje ponderado por población, como se observó con anterioridad, así mismo, existe variación del orden de ahorro porcentual si comparamos estos resultados con los obtenidos entre el escenario 1 y 2; por ejemplo, para un $50 \%$ de cobertura poblacional, el estrato 4 refiere el menor ahorro porcentual en el caso comparativo entre el escenario 1 y el escenario 2, mientras que es el estrato de mayor ahorro para el caso comparativo entre el escenario 1 y el escenario 3.

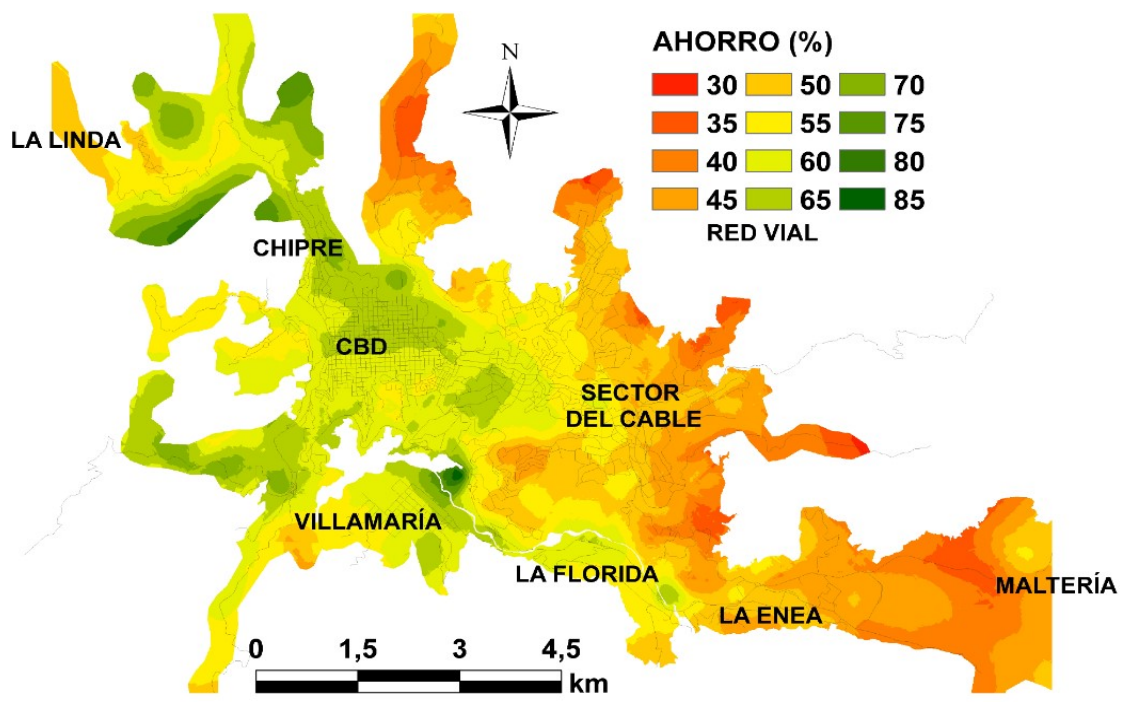

Fig. 11: Gradiente de variación temporal entre escenario 1 y escenario 3.
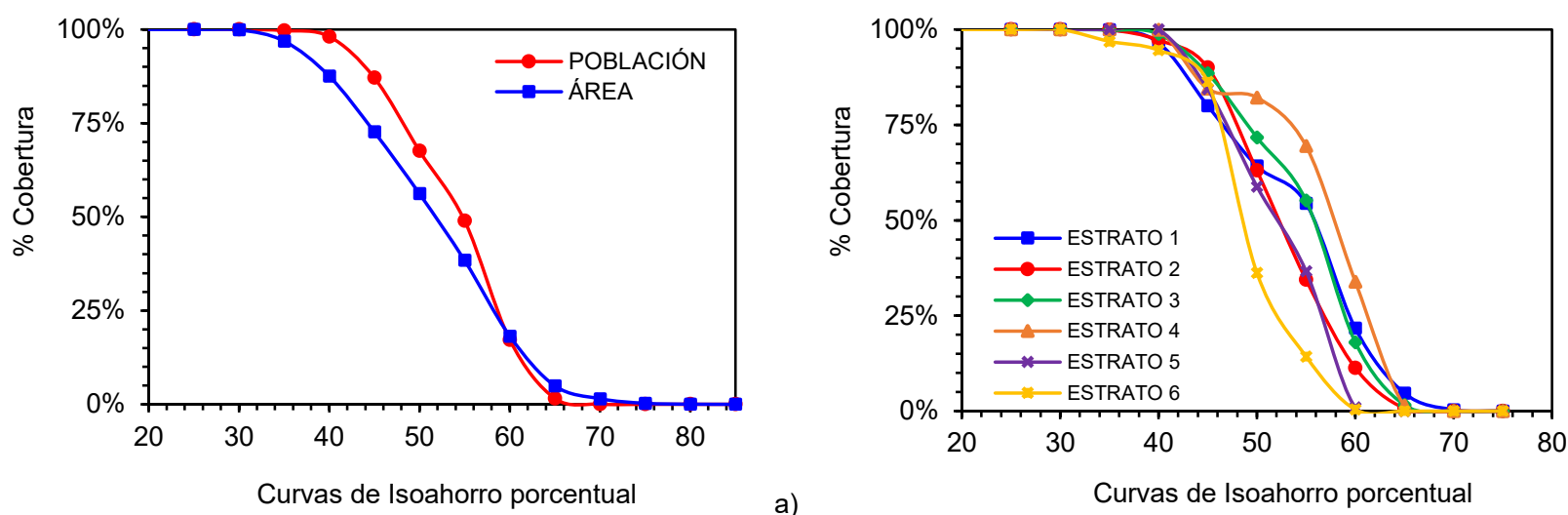

Fig. 12: Ojivas porcentuales de cobertura acumulada en el escenario 3, a) según estrato socioeconómico; b) según las variables población y área.

Ahora bien, al analizar y comparar los escenarios 2 y 3 en relación con la cobertura de los NAP (Educación, Recreación, Seguridad, Salud), se presenta en la figura 13a las curvas de tiempo medio de viaje Vs el porcentaje de cobertura de población, según estos equipamientos; en este caso se observa, por ejemplo que el escenario 3, de forma general para todos estos equipamientos, cubren de forma más rápida el total de la población de la ciudad; es decir, para el escenario 3, se cubren los NAP en tiempos medios de viaje de entre 13 y 16,5 minutos para los equipamientos salud y recreativos, respectivamente; mientras que para el escenario 2, se cubren los NAP en tiempos medios de viaje de entre 19,2 y 21,6 minutos, para nuevamente, los equipamientos salud y recreativos, respectivamente. Así mismo, en la figura 13b se presenta la relación entre las variables, porcentaje de ahorro en tiempo medio de viaje y porcentaje de cobertura poblacional según equipamiento; se observa entonces que para un $50 \%$ de cobertura poblacional, en el caso del escenario 
2 los equipamientos con mayor porcentaje de ahorro son los recreativos con un valor de $33,5 \%$, seguidos de los equipamientos educación $(33,1 \%)$, salud $(32,8 \%)$ y seguridad $(32 \%)$; en cambio, el escenario 3 refiere porcentajes de ahorro mucho más altos y en un orden diferente, salud $(56,2 \%)$, educación ( $55 \%)$, seguridad $(53,6 \%)$ y recreativos $(51 \%)$. Es decir, para el escenario 3 , el equipamiento de menor ahorro porcentual es precisamente el equipamiento de mayor ahorro porcentual para el escenario 2.
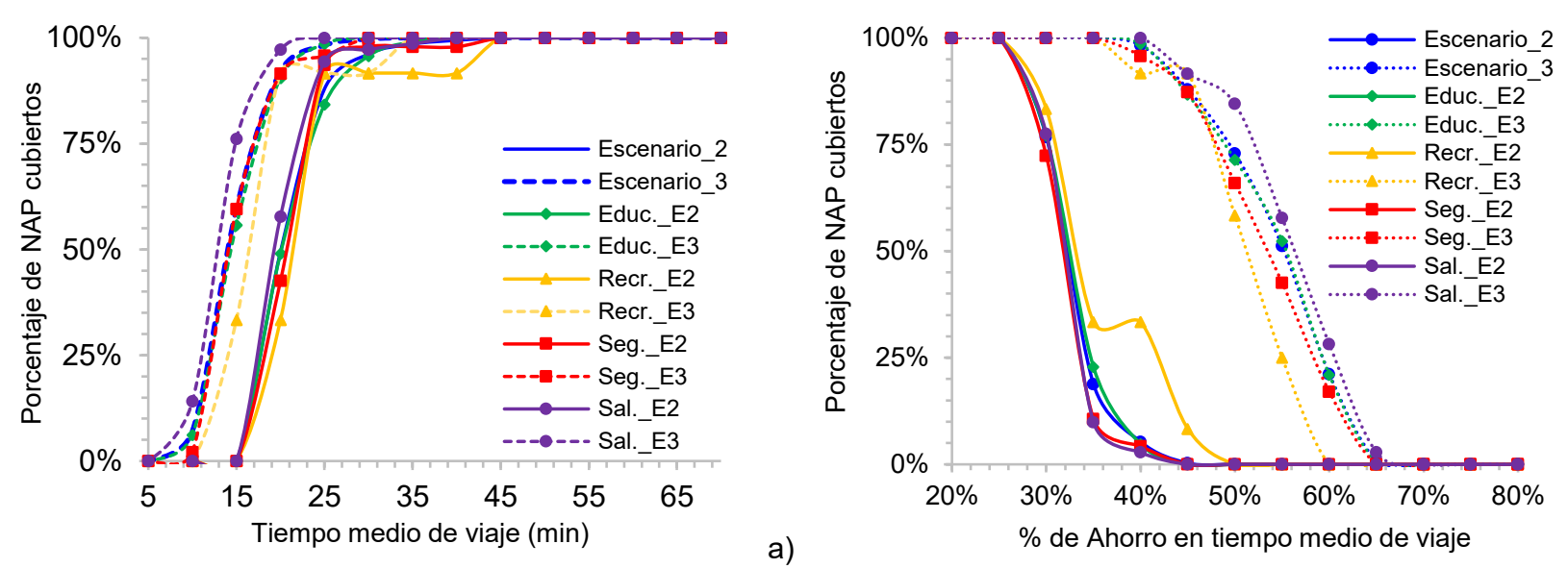

b)

Fig. 13: Porcentaje de NAP cubiertos para los escenarios 2 y 3 Vs a) tiempo medio de viaje (min), b) Porcentaje de ahorro en tiempo medio de viaje.

\section{DISCUSION FINAL}

El comportamiento de las condiciones de accesibilidad en el escenario 1, evidencia la intención, indirecta, de solventar dichas condiciones al interior de la ciudad sin considerar los diferentes sectores periféricos en los cuales reside un importante porcentaje de la población. Las intervenciones realizadas por el gobierno local en el escenario 2, facilitan la conexión de focos poblacionales importantes como La Enea (Oriente) y Villamaría (Sur), garantizando así una mejora en el tiempo de viaje para estos sectores ubicados en la periferia. El mejoramiento de las condiciones de accesibilidad para los sectores periféricos, evidencia los esfuerzos por incluir a la ciudadanía residente en las afueras a las diferentes actividades que se desarrollan en el CBD, mejorando el estilo de vida, además de garantizar el derecho a la ciudad.

El impacto en las condiciones de accesibilidad con fines de mejoramiento de la calidad de vida urbana, no solo se manifiesta en la facilidad o no de acceder a los NAP y lugares específicos dentro de esta, sino que también influyen en el número de viajes por persona que se pueden presentar en una ciudad, aumentando o disminuyendo dicho valor, dependiendo de las intervenciones infraestructurales o de gestión de movilidad que se propongan; en ciudades del primer mundo como Chicago, Londres y Tokio, se registran entre 3 y 4 viajes por persona al día (CAF, 2011), mientras que en Bogotá se registran entre 1,5 y 2 (Bocarejo y Oviedo, 2012 y en el sector de estudio de esta investigación, 2,11 viajes por persona al día (Alcaldía de Manizales, 2017).

Los resultados indican que existen importantes diferencias entre calcular la evolución de las condiciones de accesibilidad con una red de trabajo detallada (escenario 2) o con una red de trabajo simplificada (escenario 3). La red del escenario 2 fue construida de forma detallada, tanto en longitudes de vías como en características operativas, mientras que la red del escenario 3 presenta vías que están duplicadas lo que se traduce en doble direccionalidad, incluso donde en campo las vías son unidireccionales, así mismo inexistencia de vías, sobretodo de categoría local; es decir, dado el error topológico de duplicación de los arcos, se observan resultados que no son cercanos a la realidad, además de factores adicionales como la pendiente de las vías, influyen en una variable operativa como la velocidad, lo cual se traduce en resultados diferentes en términos de condiciones de accesibilidad territorial. Los efectos más directos de dichas diferencias de resultados se observarán en que los esfuerzos de impulsar infraestructuras del transporte pueden ser puestos no solo en lugares que realmente no necesitan solventar problemas tan delicados como en otros, lo cual modifica diametralmente no solo la necesidad de esfuerzos fiscales, sino las condiciones de accesibilidad futura. Lo anterior pone de manifiesto la gran importancia en términos de detalle y precisión de resultados, pues como se observó a lo largo de la investigación, el escenario 2 no sólo refiere porcentajes de ahorro porcentual mucho más reales, sino que el sector donde se deben hacer esfuerzos por mejorar las condiciones de accesibilidad es el sector occidente, sector donde precisamente, el escenario 3 establece condiciones favorables. Ç 
Después de observar y comparar los resultados de los escenarios 2 y 3 es evidente que para realizar este tipo de trabajos se debe estar contextualizado con la ciudad, su evolución en cuanto a formas de transporte y antigüedad de la infraestructura del transporte, es necesario por parte de la administración municipal que contrata este tipo de estudios, que determine con criterios serios y razonables, condiciones o requisitos de participación y que además conozca de manera previa como son las condiciones de movilidad y el funcionamiento normal de la ciudad. Se requiere como complemento al proceso de selección del consultor, contar con una supervisión (interna) o interventoría (externa), para hacer un seguimiento a los resultados y cuando sea necesario estos realicen las observaciones del caso para que éstos sean lo más precisos posible y sirvan de sustento al momento de tomar decisiones. Se resalta que la metodología en esta investigación aplicada y descrita, puede ser llevado a cabo mediante el uso de software SIG o mediante la implementación de códigos de acceso libre en software cómo $\mathrm{R}$ o Python, así mismo, las redes de trabajo base y las características operativas se pueden obtener de librerías como googlemaps, lo cual hace que el estudio sea perfectamente replicable en otras ciudades, regiones o latitudes.

\section{CONCLUSIONES}

De acuerdo al trabajo presentado y a los resultados obtenidos, se pueden plantear las siguientes conclusiones principales:

1.- Los resultados obtenidos ponen de relieve las diferencias al calcular la variación de las condiciones de accesibilidad entre el escenario 1 (año 2010) y los escenarios 2 y 3 (ambos del año 2017); por ejemplo, se evidencian disparidades importantes, lo que claramente puede direccionar los esfuerzos infraestructurales en sentidos posiblemente erróneos, y en este caso en particular, en sentidos opuesto, pues el escenario 2 claramente propone intervenir, prioritariamente, el sector occidente y el escenario 3 propondría intervenir el sector oriente.

2.- Los resultados de comparar los escenarios 1 y 2 se consideran mucho más consecuentes que los obtenidos al comparar los escenarios 1 y 3 , no sólo por los sectores de la ciudad que refieren dichos ahorros porcentuales, sino por la magnitud de los mismos, en donde para la segunda comparación se observan áreas con ahorros porcentuales de hasta un $85 \%$, lo cual con total seguridad no sucede en condiciones normales de movilidad y accesibilidad en la ciudad.

3.- Los análisis de cobertura según el tipo de NAP, muestra que para ambos escenarios (2 y 3 ), los equipamientos con mejores condiciones de accesibilidad son los de Salud, los que refieren peores condiciones de accesibilidad son los recreativos y existe diferencia entre los equipamientos seguridad y educación. No obstante, según el escenario 2, los equipamientos con mayores porcentajes de ahorro son los recreativos, mientras que son esos mismos equipamientos los que en el escenario 3 refieren el menor porcentaje de ahorro en tiempo medio de viaje; lo anterior refuerza la necesidad de tener más detalles y precisión en los análisis de accesibilidad, pues direccionarían los esfuerzos fiscales de inversión a sectores posiblemente distintos a los realmente más necesitados.

\section{AGRADECIMIENTOS}

Los autores agradecen al grupo de trabajo académico en movilidad sostenible de la Universidad Nacional de Colombia sede Manizales; así mismo, se agradece al estudiante de Doctorado en Ingeniería, Jorge Alberto Montoya Gómez por su importante colaboración en el desarrollo de la investigación.

\section{REFERENCIAS}

Alcaldía de Manizales, Plan Maestro de Movilidad de Manizales: Línea base y diagnóstico de la situación actual de la movilidad de Manizales. Manizales, (2017)

Batty, M., Accessibility: in search of a unified theory. https://doi.org/doi:10.1068/b3602ed, Environment and Planning B. Planning and Design, 36(2), 191-194 (2009)

Boarnet, M.G., y Wang, X., Urban spatial structure and the potential for vehicle miles traveled reduction: The effects of accessibility to jobs within and beyond employment sub-centers. https://doi.org/10.1007/s00168-019-00900-7, The Annals of Regional Science, 62, 381-404 (2019)

Bocarejo, J.P., y Oviedo, D.R., Transport accessibility and social inequities: a tool for identification of mobility needs and evaluation of transport investments. https://doi.org/10.1016/j.jtrangeo.2011.12.004, Journal of Transport Geography, 24, 142-154 (2012)

CAF (2011). Desarrollo urbano y movilidad en América Latina, Caracas: Corporación Andina de Fomento.

Cardona, S., Propuesta metodológica para el cálculo de las penalidades por giro en modelos de accesibilidad, Tesis de Maestría, Universidad Nacional de Colombia, Manizales, Colombia (2018) 
Carrion, C., y Levinson, D., Over- and Under-Estimation of Travel Time on Commute Trips: GPS vs. Self-Reporting. https://doi.org/10.3390/urbansci3030070, Urban Science, 3, 70 (2019)

Conesa, A., The accessibility assessment and the regional range of transit-oriented development: An application of schedule accessibility measures in the Nord Pas-de-Calais region. https://doi.org/10.5198/jtlu.2018.850, Journal of Transport and Land Use, 11(1), 119-141 (2018)

Cui, M., y Levinson, D., Full cost accessibility. https://doi.org/10.5198/jtlu.2018.1042, Journal of Transport and Land Use, 11(1), 661-679 (2018)

Escobar, D.A., Cadena, C., y Salas, A., Cobertura Geoespacial de nodos de actividad primaria. Análisis de los aportes a la sostenibilidad urbana mediante un estudio de accesibilidad territorial. doi:10.14508/reia.2015.12.23.13-27, Revista EIA 12(23), 13-27 (2015a)

Escobar, D.A., Montoya, J.A. y Sabogal, O.A., Dónde ubicar nuevos nodos comerciales en la ciudad de Manizales Colombia? Aplicación de un análisis geográfico, Revista Espacios, 38(53), 7 (2017)

Escobar, D.A., Tapasco, O.A. y Giraldo, J.A., Medición de Desempeño del Sistema de Transporte Cable Aéreo de la Ciudad de Manizales en Colombia, usando Tres Enfoques: Analítico, Simulado y de Accesibilidad Urbana. https://doi.org/10.4067/S0718-07642015000600020, Información Tecnológica, 26(6), 199-210 (2015b)

Geurs, K.T. y Van Wee, B., Accessibility evaluation of land-use and transport strategies: review and research directions. https://doi.org/10.1016/j.jtrangeo.2003.10.005, Journal of Transport Geography, 12(2), 127-140 (2004)

Gonzales, J., Guasch, J. y Serebrisky, T., Latin America: Addressing high logistics costs and poor infrastructure for merchandise transportation and trade facilitation, The World Bank (2007)

Graser, A. y Dragaschnig, M., Open Geospatial Tools for Movement Data Exploration. https://doi.org/10.1007/s42489-02000039-y, KN - Journal of Cartography and Geographic Information, (2020)

Hansen, W., How accessibility shapes land use. https://doi.org/10.1080/01944365908978307, Journal of the American Institute of Planners, 25(2), 73-76 (1959)

Liu, S. y Zhu, X., An integrated GIS approach to accessibility analysis. https://doi.org/10.1111/j.1467-9671.2004.00167.x, Transactions in GIS, 8(1), 45-62 (2004)

Long, J.A., Weibel, R., Dodge, S. y Laube, P., Moving ahead with computational movement analysis. https://doi.org/10.1080/13658816.2018.1442974, International Journal of Geographical Information Science, 32(7), 1275$1281(2018)$

Moncada, C.A., Cardona, S. y Escobar, D.A., Saving Travel Time as an Urban Planning Instrument. Case Study: Manizales, Colombia. https://doi.org/10.5539/mas.v12n6p44, Modern Applied Science, 12(6), 44-57 (2018)

Montoya, J.A., Escobar, D.A. y Moncada, C.A., Propuesta de ubicación de nuevos centros comerciales, aplicación de un análisis de accesibilidad territorial urbana, Revista Espacios, 38(51), 4 (2017)

Perilla, D.J., Escobar, D.A. y Cardona, S., New transportation infrastructure impact in terms of global average access intersection la Carola, Manizales (Colombia) case study. https://doi.org/10.12988/ces.2018.812, Contemporary Engineering Sciences, 11 (5), 215-227 (2018)

Sahebgharani, A., Haghshenas, H. y Mohammadi, M., A Markovian measure for evaluating accessibility to urban opportunities. https://doi.org/10.5198/jtlu.2019.1408, Journal of Transport and Land Use, 12(1), 19-43 (2019)

Sarmiento, O.L., Higuera, D. y otros veinte autores, Urban Transformations and Health: Methods for TrUST - a Natural Experiment Evaluating the Impacts of a Mass Transit Cable Car in Bogotá, Colombia. https://doi.org/10.3389/fpubh.2020.00064, Frontiers in Public Health, 8(64), 1-15 (2020)

Urrunaga, R. y Aparicio, C., Infraestructura y crecimiento económico en el Perú, Revista CEPAL. 107, 157-177 (2012)

Vega, A., A multi-modal approach to sustainable accessibility in Galway. https://doi.org/10.1080/20429843.2011.9727923, Regional Insights, 2(2), 15-17 (2011)

Wachs, M. y Kumagai, T.G., Physical accessibility as a social indicator. https://doi.org/doi:10.1016/0038-0121(73)900414, Socio-Economic Planning Sciences, 7(5), 437-456 (1973)

Weber, J., The properties of topological network connectivity measures and their application to U.S. urban freeway networks. https://doi.org/10.1080/00330124.2015.1106324, The Professional Geographer, 68(3), 485-495 (2016)

Weiss, D.J., Nelson A. y otros veinte autores, A global map of travel time to cities to assess inequalities in accessibility in 2015. https://doi.org/10.1038/nature25181, Nature, 553, 333-336 (2018)

Zuluaga, J.D., y Escobar, D.A., Geomarketing Analysis for Shopping Malls in Manizales (Colombia). Accessibility approach methodology, Revista Espacios, 38 (21), 20 (2016) 